\title{
Spectroscopic, Kinetic Studies of Polyaniline-Flyash Composite
}

\author{
Raju Khan, Puja Khare, Bimala Prasad Baruah, Ajit Kumar Hazarika, Nibaran Chandra Dey \\ North East Institute of Science \& Technology, Council of Scientific \& Industrial Research, Jorhat, India \\ E-mail: khan.raju@gmail.com,rajukhan@rrljorhat.res.in \\ Received December 21, 2010; revised February 2, 2011; accepted March 3, 2011
}

\begin{abstract}
Polyaniline-fly ash (PANI-FA) composites were prepared by oxidative polymerization of aniline with fly ash in presence of ammonium persulphate (APS). The PANI-FA composites were prepared with different concentrations of fly ash to aniline ratio. The composites, so prepared, were characterized by UV-vis spectroscopy, Fourier transform infrared (FTIR) spectroscopy, scanning electron microscopy (SEM) and thermogravimetric analysis (TGA). The thermal stability was studied by TGA and total weight loss of PANI, FA and PANI-FA composites having FA composition of $0.02 \%, 0.1 \%, 0.5 \%$ and $1.0 \%$ were found to be $82 \%, 39 \%$, $67 \% 65 \%, 62 \%$ and $61 \%$, respectively. The UV-vis spectroscopy of the PANI-FA polymeric composite shows absorption maxima at 315 and $350 \mathrm{~nm}$ (due to $\pi-\pi^{*}$ transition of the benzenoid rings), and $578-712 \mathrm{~nm}$ (due to charge transfer excitations of the quinoid structure), which are characteristic of emeraldine base. FTIR spectra of the PANI-FA composite is similar to that of pure polyaniline (PANI) but with the bands for $\mathrm{C}=\mathrm{N}, \mathrm{C}=\mathrm{C}$ and $\mathrm{C}-\mathrm{N}$ shifted to lower wave numbers, i.e., $1585,1494,1327$ and $1113 \mathrm{~cm}^{-1}$ due to strong interaction of $\mathrm{Fe}_{2} \mathrm{O}_{3}$ and PANI matrix. SEM shows the complexation of metal oxide with emaraldine base of PANI, significantly changing the aggregate state of polymeric molecular chain.
\end{abstract}

Keywords: Polyaniline, Fly-Ash, TGA, UV-Vis Spectroscopy, FT-IR, Kinetic Study and SEM

\section{Introduction}

Polyaniline (PANI) can be synthesized chemically or electrochemically in an acidic medium. For chemical synthesis, an appropriate oxidant is required. There are three forms of PANI, namely fully oxidized pernigraniline, half-oxidized emeraldine base (EB) and fully reduced leucoemeraldine base (LB). Emeraldine is said to be the most stable form of PANI and also the most conductive form when doped (emeraldine salt) [1]. PANI is a versatile substance which has potential applications in corrosion prevention, as sensors, in electronics and electrochromic devices, and in batteries [2-6]. Conducting polymers are attractive alternative to conventional inorganic gas sensors due to potentially lower costing, simpler packing and compatibility with flexible substrates $[7,8]$. Polyaniline (PANI), with unique electrical and optical properties, is a promising candidate for wide range of potential applications $[9,10]$. The changes in electrical and optical properties of PANI induced through interaction of oxidizing or reducing agents make it suitable for sensing applications [11-16]. However, the processing difficulty and poor stability due to polymer degradation, have to be overcome for its successful applications.

Furthermore, the developments of organic-iorganic nanocomposites are expected to play an important role as gas sensing materials. Efforts are continuing to engineer organic-inorganic materials with enhanced physical and optical properties for gas sensing applications. Nanocomposite thin films of polymer-inorganic nanoparticles are reported to have porous morphology caused by solid-state polymerization [17]. This porous morphology has added advantage for sensing application due to enhanced penetration of bio-molecules into the polymer matrix.

North East India produces a large quantity of fly ash from its coal-fired power plant. In India, substantial part of electric power (about 65\%) is generated from coal or lignite fired thermal power stations. One of the major pollutants generated in a coal based thermal power plant is flyash. Silica $\left(\mathrm{SiO}_{2}\right)$, alumina $\left(\mathrm{Al}_{2} \mathrm{O}_{3}\right)$, iron oxide $\left(\mathrm{Fe}_{2} \mathrm{O}_{3}\right)$ and titanium oxide $\left(\mathrm{TiO}_{2}\right)$ are major constituents of FA. These metal oxides have been used in one way or 
the other in the preparation of nanocomposites. For example, $\mathrm{Al}_{2} \mathrm{O}_{3}$ and $\mathrm{Fe}_{2} \mathrm{O}_{3}$ have been used as catalyst support for the production of organic-inorganic porous nanocomposites. In this paper we are using natural composition of metal oxides for the preparation of PANI-FA composite, which forms morphologically porous marix and have improved thermal stabilty. The incorporation of FA into the polymeric network introduces uniform porosity and is expected to be advantageous for gas sensing and biosensing applications.

\section{Experimental}

\subsection{Chemical and Reagents}

Aniline (25\% v/v in water), acetone, ammonium persulphate, dimethylformamide (DMF) and hydrochloric acid were all products of Sigma-Aldrich Chemicals. Only freshly vacuum-distilled aniline was used for the synthesis of PANI. EDX study showed that the main constituents of the FA used in this study are as follows: silica as $\mathrm{SiO}_{2} \sim 50 \%$, alumina as $\mathrm{Al}_{2} \mathrm{O}_{3} \sim 30 \%$, calcium oxide as $\mathrm{CaO} \sim 5 \%$, iron oxide as $\mathrm{Fe}_{2} \mathrm{O}_{3} \sim 4 \%$, magnesium oxide as $\mathrm{MgO} \sim 2 \%$ and titanium oxide as $\mathrm{TiO}_{2} \sim 2 \%$.

\subsection{Synthesis of Polyaniline-Fly Ash Composites}

For preparation of Polyaniline-Fly Ash (PANI-FA) composite, the procedure for the synthesis of composite was followed [18]. 0.1 M aniline was prepared in $2 \mathrm{M}$ $\mathrm{HCl}$ solution. $100 \mathrm{~mL}$ of this aniline solution was stirred and FA was added in the solution with vigorous stirring $(0 \%, 10 \%, 20 \%, 30 \%$, and $40 \%$ FA to aniline $\mathrm{w} / \mathrm{w}$ ratio). The mixture was kept in an ice bath with continuous stirring. Then $0.1 \mathrm{~mol}$ of ammonium persulphate (APS) was added slowly to the aniline solution until the reaction mixture turned green. The reaction mixture was then stirred for $8 \mathrm{~h}$. The product was collected by filtration and washed with water and acetone until the washing was colourless. The collected samples were dried at room temperature and preserved for further studies.

\subsection{Characterization of PANI, FA and PANI-FA Composites}

All the UV-vis absorbance experiments were performed at room temperature with UV-vis Specord-200 using quartz cuvettes. The UV-vis sample solution was prepared by dissolving $0.005 \mathrm{~g}$ of a PANI composite in $10 \mathrm{~mL}$ dimethylformamide (DMF) and experimental wavelength scanned between 200 to $800 \mathrm{~nm}$. The infrared spectra of the PANI, FA and PANI-FA polymer composite were recorded on Perkin Elmer system 2000 FT-IR spectrometer using $\mathrm{KBr}$ pallets at room tempera- ture in the region of 3600-4000 $\mathrm{cm}^{-1}$. Scanning electron microscopy (SEM) analysis was performed with a Hitachi X-650 scanning electron microanalyser which has an operating voltage window of $5-40 \mathrm{kV}$. Micrographs were obtained for samples of the composites mounted on aluminium stubs using conductive glue and coated with a thin layer of gold. Experiments were carried out in a Leco TGA 701 thermal analysis system with 0.5 gm each of fly-ash samples in a stream of nitrogen with a flow rate of $40 \mathrm{~mL} \cdot \mathrm{min}^{-1}$ and linear heating rate of $10^{\circ} \mathrm{C} \cdot \mathrm{min}^{-1}$. The weight loss (thermogravimetric TG signals) and the rate of weight loss (differential thermogravimetric DTG signals) as a function of time or temperature were recorded.

\section{Results and Discussion}

\subsection{FTIR Properties}

FTIR spectra of PANI, FA \& PANI-FA composite are shown in Figure 1. FTIR band at $2982-3463 \mathrm{~cm}^{-1}$ corresponds to $\mathrm{N}-\mathrm{H}$ stretching with hydrogen bonded amino groups and free $\mathrm{O}-\mathrm{H}$ stretching vibration and is attributed to the N-H stretching vibrations of the leucoemeraldine component. The characteristic absorption band observed for PANI-flyash at $3463 \mathrm{~cm}^{-1}$ and $1690 \mathrm{~cm}^{-1}$ are assigned to the N-H stretching vibration mode, and $\mathrm{NH}_{2}$ deformation in aniline unit respectively. The absorption band $2925 \mathrm{~cm}^{-1}$ and $2830 \mathrm{~cm}^{-1}$ are assigned to the aromatic $\mathrm{sp}^{2}$ hybridized $\mathrm{C}-\mathrm{H}$ stretching vibration mode and aliphatic hydrocarbon $\mathrm{C}-\mathrm{H}$ stretching due to $-\mathrm{CH}_{2}$ - bonded with aniline unit. Strong peak at $2355 \mathrm{~cm}^{-1}$ was associated with $-\mathrm{N} \equiv \mathrm{N}$ in diazonium salts. The absorption bands ob-

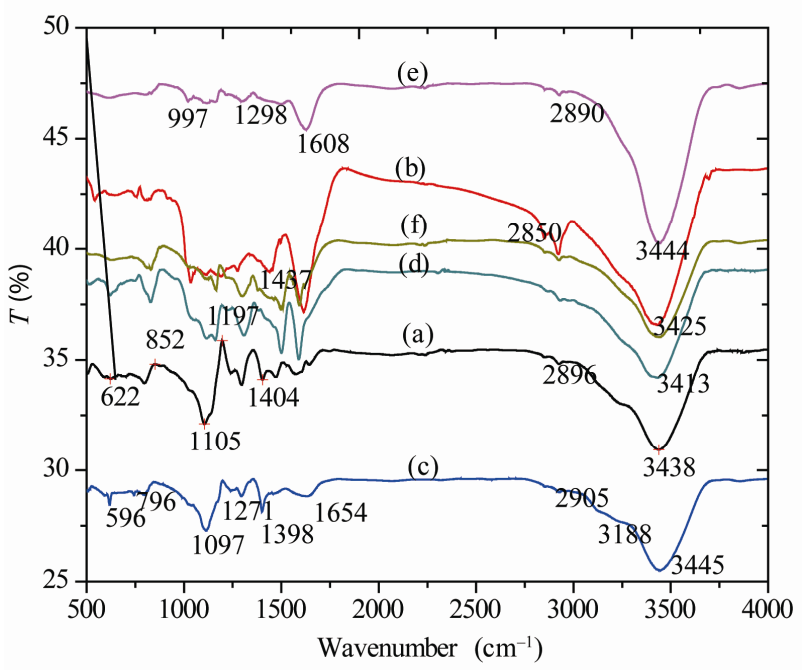

Figure 1. FTIR spectra of chemically synthesized of (a) PANI; (b) FA; (c) and composite of polyaniline-flyash, PANI-FA (0.02); (d) PANI-FA (0.1); (e) PANI-FA (0.5) and (f) PANI-FA (1.0). 
served at 1567 and $1482 \mathrm{~cm}^{-1}$ in PANI is assigned respectively to the non-symmetric vibration mode of $\mathrm{C}=\mathrm{C}$ in quinoid and benzenoid ring system in polyaniline. The C-N stretching vibration mode in aromatic amine nitrogen (quinoid system) in doped polyaniline is found at $1290 \mathrm{~cm}^{-1}$, corresponding to the oxidation or protonation state. The absorbance peak at $1233 \mathrm{~cm}^{-1}$ is attributed to $\mathrm{C}-\mathrm{N}$ stretching vibration mode in benzenoid ring system of polyaniline due to the conducting protonated form. In plane vibration of $\mathrm{C}-\mathrm{H}$ bending mode in $\mathrm{N}=\mathrm{Q}=\mathrm{N}$, $\mathrm{Q}-\mathrm{N}^{+} \mathrm{H}-\mathrm{B}$ or $\mathrm{B}-\mathrm{N}^{+} \mathrm{H}-\mathrm{B}$ (where $\mathrm{Q}=$ quinoid and $\mathrm{B}=$ benzenoid) is observed at $1146 \mathrm{~cm}^{-1}$. The presence of this absorption band is expected due to the polymerization of PANI, i.e., polar structure of the conducting protonated form. The absorption bands at 874 and $799 \mathrm{~cm}^{-1}$ are attributed to the aromatic ring and out of plane $\mathrm{C}-\mathrm{H}$ deformation vibrations for 1, 4-disubstituted aromatic ring system[19].

In FTIR spectrum of purified FA, the characteristic $3426 \mathrm{~cm}^{-1}$ band indicating the stretching vibration of the -OH group appears due to some components with an -OH group or crystal lattice water on the surface of FA. Broadening in characteristic peaks range 2750 - 3463 $\mathrm{cm}^{-1}$ is associated with $\mathrm{N}-\mathrm{H}$ stretching vibration of PANI. The decrease in broadening of FTIR bands in the range $2750-3463 \mathrm{~cm}^{-1}$ was due to covalent and hydrogen bonding between $-\mathrm{NH}_{2}$ and $-\mathrm{OH}$ group of PANI and FA respectively. The very sharp FTIR peak at 1632 $\mathrm{cm}^{-1}$ was associated with $\mathrm{C}=\mathrm{O}$ stretch in $-\mathrm{HNCOCH}_{3}$ group of PANI-FA matrix. The IR spectra of PANI composite in presence of FA exhibit new absorption peaks distinctly at 1522, 1447, 1280, 1196 and $641 \mathrm{~cm}^{-1}$ which could be assigned to the presence of various metal oxides in the composite. The peak around $1113 \mathrm{~cm}^{-1}$ may be attributed to the presence of silica within the composite. The FTIR peaks at $1030 \mathrm{~cm}^{-1}$ for the FA corresponds to the internal $\mathrm{SiO}_{4}$ tetrahedra, especially the $\mathrm{Si}-\mathrm{O}-\mathrm{Si}$ chain structure. The peaks at $1030-1196 \mathrm{~cm}^{-1}$ of the FA correspond to a cyclic $\mathrm{Si}-\mathrm{O}-\mathrm{Si}$ structure. The FA indicates the FTIR spectra of $\mathrm{Fe}_{2} \mathrm{O}_{3}$, where the bands around 540-466 $\mathrm{cm}^{-1}$ are assigned to $\mathrm{Fe}-\mathrm{O}$ stretch. It can be seen that the FTIR spectra of the PANI-FA composite is similar to that of pure PANI where the bands for $\mathrm{C}=\mathrm{N}$, $\mathrm{C}=\mathrm{C}$ and $\mathrm{C}-\mathrm{N}$ are all shifted to lower wave numbers, i.e. $1585,1494,1327$ and $1113 \mathrm{~cm}^{-1}$ due to strong interaction of $\mathrm{Fe}_{2} \mathrm{O}_{3}$ and PANI [20].

\subsection{UV-Vis Properties}

The UV-vis absorption spectra of PANI and PANI-FA composite are shown in Figure 2. Two absorption bands are observed in the wavelength region from 315 to $350 \mathrm{~nm}$ and a small band at 578 to $712 \mathrm{~nm}$ for the PANI (Figure 2(b)). PANI always exhibits a $\pi-\pi^{*}$ transition,

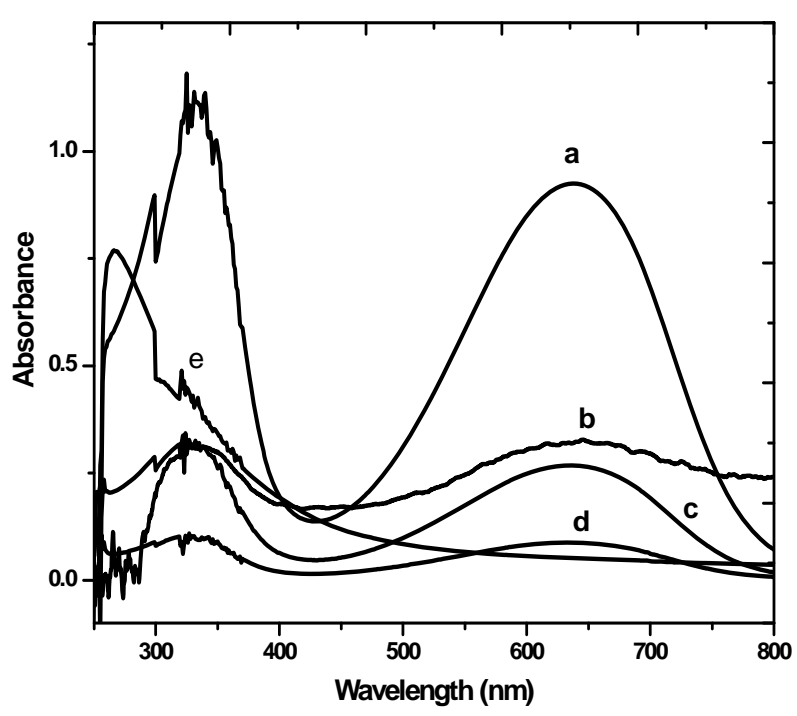

Figure 2. UV-vis spectra of chemically synthesized of (a) PANI-FA (1.0), (b) PANI, (c) PANI-FA (0.5), (d) PANI-FA (0.1) and (e) FA.

usually closer to $315 \mathrm{~nm}$ [21]. Partially oxidized PANI and its oligomers display an additional absorption at around $712 \mathrm{~nm}$ associated with the quinoid (oxidized) units [22]. These peaks are characteristic of the PANI emeraldine base $[23,24]$ and indicate that nanostructured PANI composites are stabilized in the emeraldine base redox state. The peak at $315 \mathrm{~nm}$ is attributed to $\pi-\pi^{*}$ transition of benzoid rings and the peak at $712 \mathrm{~nm}$ is attributed to the charge transfer excitation of the quinoid structure. In the spectra of pure FA, peaks are observed in the regions at 258-289 $\mathrm{nm}$ but were found absent in the region 600-700 nm (Figure 2(e)). PANI-FA prepared without aging show clear similarity in their UV-vis spectra particularly with the complete absence of the absorption maxima at 320 and $630 \mathrm{~nm}$ which is associated with the stabilization of the composite in the emeraldine form. Comparatively, PANI-FA composite show clear similarity in their UV-vis spectra particularly with the presence of the absorption maxima at 315 and 610 $\mathrm{nm}$ which is associated with the stabilization of the composite in the emeraldine form. Comparison of the PANI and PANI-FA composite spectra shows that FA stabilizes the polyanilines in its emeraldine form.

\subsection{TGA Properties}

TGA is widely used to study all physical process involving the weight changes such as to measure the diffusion characteristics and the moisture uptake of a sample. The thermo gravimetric profiles of PANI, FA and PANI-FA polymer composite is shown in Figure 3. Total weight loss of PANI, FA and PANI-FA polymer composite PANI-FA (0.02), PANI-FA (0.1), PANI-FA (0.5) and 


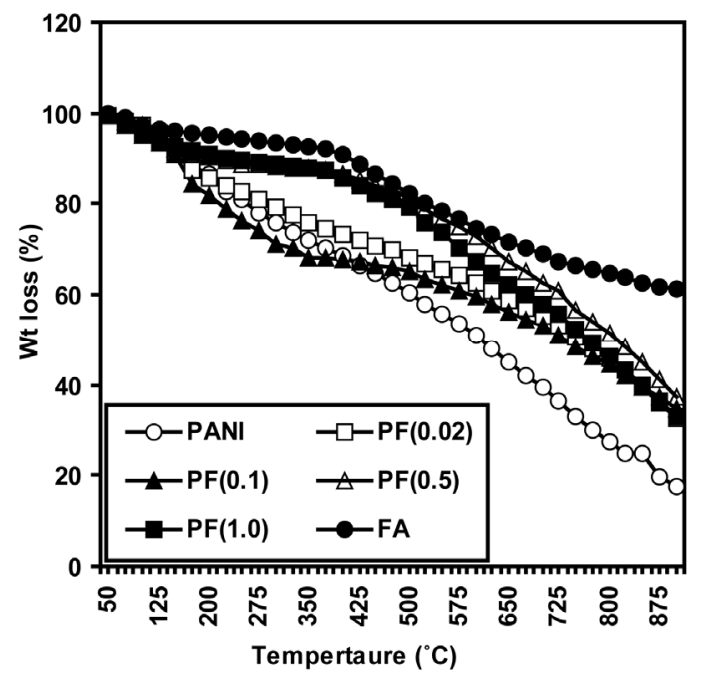

(a)

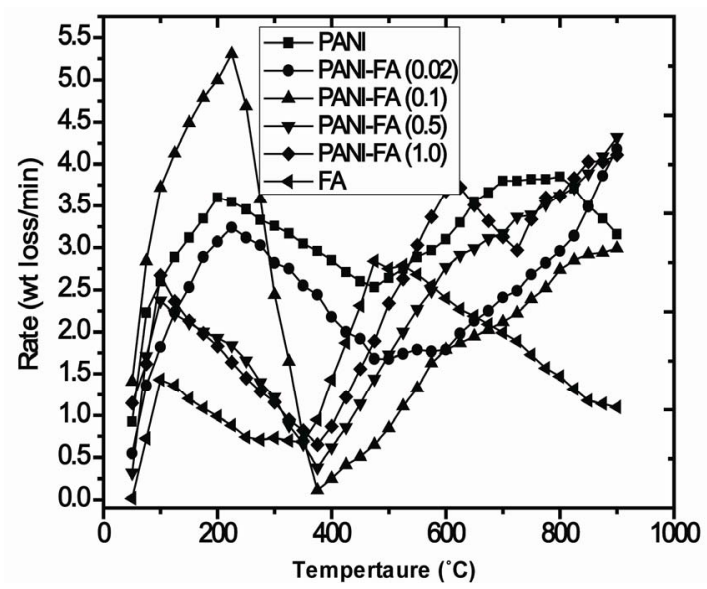

(b)

Figure 3. Thermo gravimetric analysis (TGA) of chemically synthesized of (Figure 3(a)) and DTG of (Figure 3(b)) PANI, FA and composite of polyaniline-flyash PANI-FA (0.02), PANI-FA (0.1) PANI-FA (0.5) and PANI-FA (1.0).

PANI-FA (1.0) are 82\%, 39\%, 67\%, 65\%, 62\% and $61 \%$, respectively. Decrease in ultimate weight loss indicates the interaction of PANI with the metal oxides such as $\mathrm{Al}_{2} \mathrm{O}_{3}$, Ti-O-Ti and $\mathrm{SiO}_{2}$ present in FA. FTIR spectra of these composites also show strong interaction of $\mathrm{SiO}_{2}$, $\mathrm{Fe}_{2} \mathrm{O}_{3}$ and PANI. This may be due to the Van der-Walls binding of PANI-FA polymer composites.

The thermogram of PANI indicates three major stages of weight loss. In the first stage, $3 \%-4 \%$ weight loss at temperature up to $125^{\circ} \mathrm{C}$ is associated with the loss of water molecules from the polymer matrix [1]. The weight loss at second stage that commences after $125^{\circ} \mathrm{C}$ until $225^{\circ} \mathrm{C}$ (about $9-12 \%$ ) is due to the removal of the acid dopant bound to the polyaniline chain and low molecular weight oligomers. A slow and somewhat gradual weight loss profile observed starting at $225^{\circ} \mathrm{C}$ onward, represent degradation of the skeletal polyaniline chain structure after the dopant has been removed [25]. Above $600^{\circ} \mathrm{C}$, the results obtained are associated with the residues only. The thermogram of fly ash indicates four major stages of weight loss (Figure 3(b)). PANI and composite PANIFA(0.02) and PANI-FA(0.1) exhibit similar pattern, with a small variation in degradation temperature, while thermograms of PANI-FA(0.5) and PANI-FA (1.0) show similar pattern with FA. In the first stage, 3-4\% weight loss at temperature up to $125^{\circ} \mathrm{C}$ is associated with the loss of water molecules [26]. In the second stage corresponding to temperature zone $125^{\circ}-400^{\circ} \mathrm{C}$, the weight loss (about $9-12 \%$ ) is due to the evolution of thermally labile compounds and the breaking of aliphatic structures with low dissociation bonds in the carbonaceous matrix of FA. In the third stage that commences after $400^{\circ} \mathrm{C}$ until $700^{\circ} \mathrm{C}$, maximum weight loss occurs $(21 \%)$ due to release of shoot particles attached with $\mathrm{SiO}_{2}$ system of FA $[9,16]$. Above, $700^{\circ} \mathrm{C}$ gradual decreases were observed due to thermal degradation of mineral matter of fly ash. The weight loss of PANI, fly-ash and compositions of PANI-FA (0.02), PANI-FA (0.1), PANI-FA (0.5) PANI-FA (1.0) have been found $82 \%, 38.9 \%, 67 \% 65 \%$, $62 \%$ and $61 \%$, respectively. A decrease in weight loss of composite is observed with the increasing FA content. It indicates the interaction of polyaniline with mineral matter of the FA. As indicated earlier, $\mathrm{SiO}_{2}, \mathrm{Fe}_{2} \mathrm{O}_{3}$ and $\mathrm{Al}_{2} \mathrm{O}_{3}$ are the major oxides present in the FA. FTIR spectra of these composites confirm strong interactions with metal oxides $\mathrm{SiO}_{2}, \mathrm{Fe}_{2} \mathrm{O}_{3}$ and polyaniline in the composite matrix.

\subsection{Kinetic Properties}

The activation energies of PANI, FA and PANI-FA composites were determined by the integral method [27]. It is assumed a first order reaction [28,29]. Applying Arrhenius equation for reactions, it can be expressed as

$$
\mathrm{d} x / \mathrm{d} t=A \exp (-E / R T)(1-x)
$$

where $A$ is pre-exponential factor, $E$ is the activation energy, $T$ is temperature in $K, R$ is gas constant, $t$ is time and $x$ is weight loss fraction or decomposition during pyrolysis which can be calculated by

$$
x=\left(W_{0}-W_{t}\right) /\left(W_{0}-W_{f}\right)
$$

where $W_{0}$ is the original mass of the test sample, $W_{t}$ is the mass at time $t$ or at temperature $T$ and $W_{f}$ is the final mass at the end of pyrolysis.

The constant heating rate during pyrolysis is $H=\mathrm{d} T / \mathrm{d} t$ for $H$ being the heating rate. Rearranging the Equation (1) and integration gives

$$
\ln \left[-\ln (1-x) / T^{2}\right]=\ln [A R / H E(1-2 R T / E)]-E / R T
$$


The expression $\ln \{A R[1-(2 R T / E)]\} / H E$ in Equation (3) is essentially constant for most of the values of $E$ and temperature range of the pyrolysis. By plotting the left side of Equation (3) against $1 / T$, a straight line is obtained indicating the process to be of first order reaction (Figure 4). From the slope, $-E / R$, the activation energy $E$ can be determined. Pre-exponential factor $A$ was determined by substituting the $T$ in the intercept (Equation (3)) with temperature at which

$W_{t}=\left(W_{0}+W_{f}\right) / 2$. From DTG (Figure 3(b)) of PANI, FA and PANI-FA composite, it could be seen that the reactions was not described by one first order reaction but could be described in two consecutive first order reactions instead. Equation (3) is applied separately to each stage. The conversion of $x$ was recalculated for each reaction. From the slope of each line, the value of $E$ can be obtained for different stages (Table 1). DTG curve of PANI and FA show two peaks. The first peak corresponds to devolatilization of PANI and FA. Therefore, the second one corresponds to thermal degradation of residue of PANI and mineral matter of FA, respectively. The overall, DTG curve shapes of PANI and its composites are similar to a combination curve of its components indicating that PANI is a dominant factor affecting the thermal stability of the composite. The kinetic energy of first peak of composite shift to higher sites with increasing FA content in composites. The reaction with higher activation energy means that the reaction needs more energy from the surroundings. It indicates that the composites are thermally more stable as compared to PANI and FA. The kinetic energy of second peak of composite was in between that of PANI and FA and also shift to higher site with increasing FA content in the composite. These facts indicate that some intimate interaction among skeleton of PANI and mineral matter content of FA makes the kinetic energy higher than that of PANI itself.

\subsection{Scanning Electron Microscopy}

Scanning electron microscopy (SEM) in Figure $\mathbf{5}$ is showing the general features of the original fly ash (Figure 5(a)). As it can be seen in the figure, the fly ash is mainly constituted by compact or hollowed spheres but with a regular smooth texture. Often, on the surface of spheres the existence of solid deposits or small crystals could be observed which could be soluble alkaline sulphates, dendritic shaped particles of iron minerals, mullite crystals (Figures 5(g-i)) etc. Also, some quartz particles, residue of un-burnt coal or some vitreous unshaped fragments could be seen. SEM of PANI shows porous, non-uniform structure (Figure 5(b)). PANI-FA composite shows (Figures 5(c)-(f)) the formation of rough micro structure having uniformly distributed metal oxide embedded in the PANI-FA polymer matrix. The concentration of increasing FA in polymer composite is found to play an important role in the surface morphology. The complexation of metal oxide with emaral

Table 1. Activation energy (KJ/Mol) of PANI, FA, and PANI-FA composite during pyrolysis.

\begin{tabular}{ccccccc}
\hline $\begin{array}{c}\text { Temperature } \\
\left({ }^{\circ} \mathrm{C}\right)\end{array}$ & PANI & FA & $\begin{array}{c}\text { PANI- } \\
\text { FA (0.02) }\end{array}$ & $\begin{array}{c}\text { PANI- } \\
\text { FA (0.1) }\end{array}$ & $\begin{array}{c}\text { PANI- } \\
\text { FA(0.5) }\end{array}$ & $\begin{array}{c}\text { PANI- } \\
\text { FA(1.0) }\end{array}$ \\
\hline $50-400$ & 80.39 & 80.23 & 84.23 & 88.24 & 97.29 & 99.23 \\
$500-800$ & 47.30 & 68.50 & 48.83 & 50.08 & 53.45 & 57.08 \\
\hline
\end{tabular}
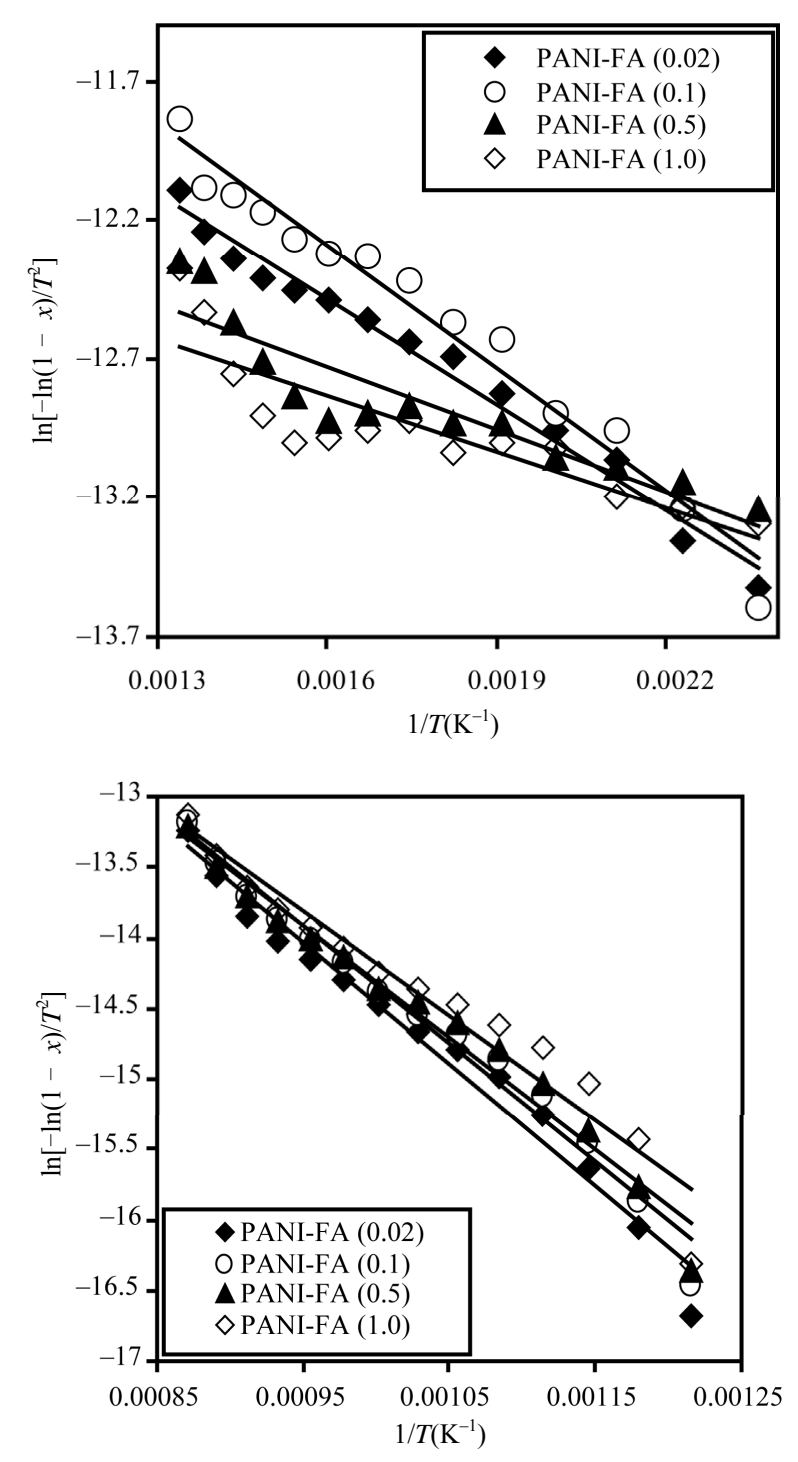

Figure 4. The activation energies of PANI-FA composites as determined by the integral method. 


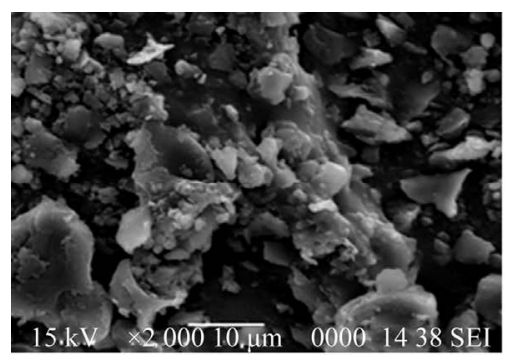

(a)

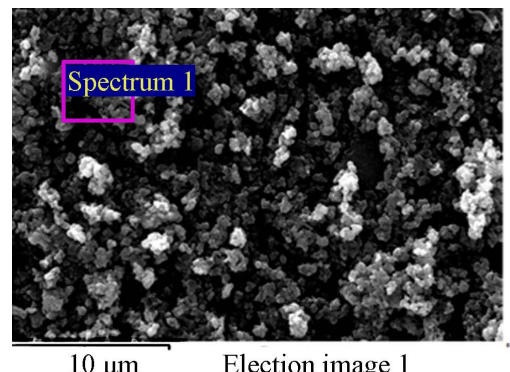

(d)

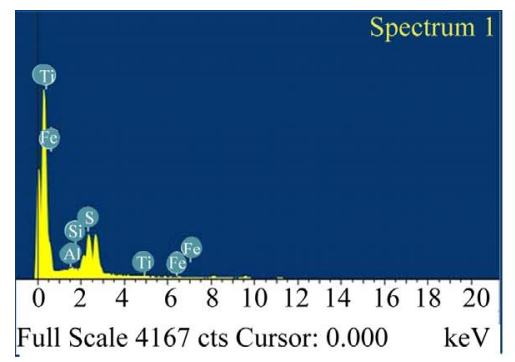

(g)

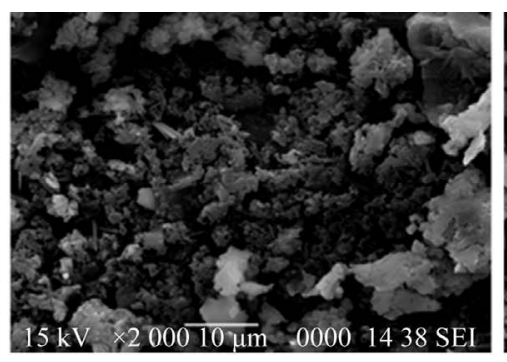

(b)

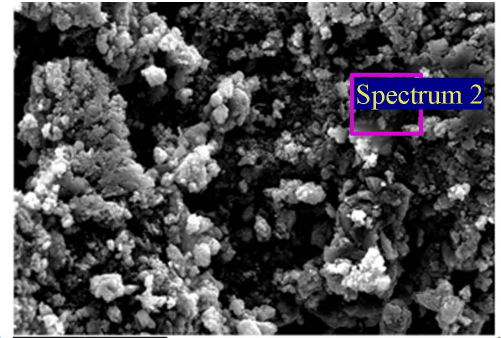

$10 \mu \mathrm{m}$

Election image 1

(e)

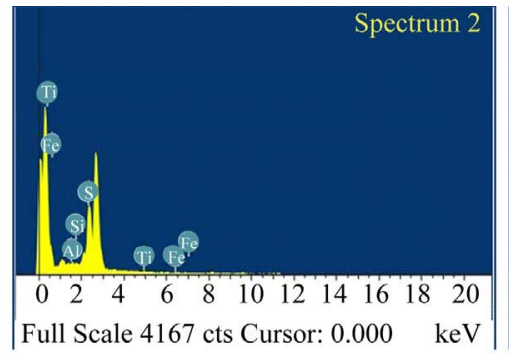

(h)

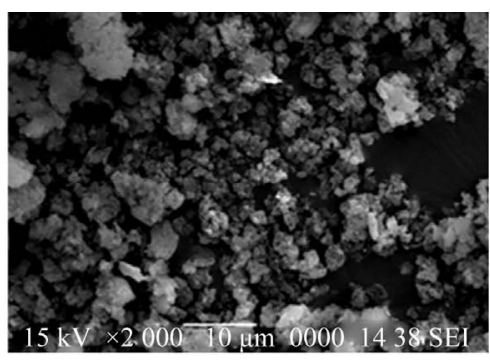

(c)

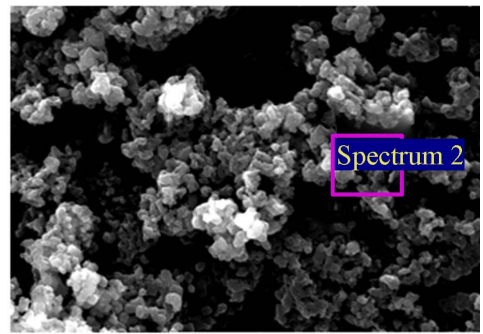

Election image 1

(f)

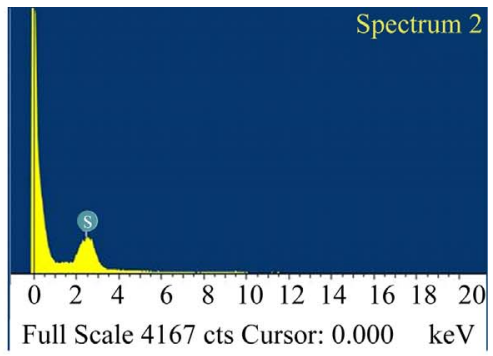

(i)

Figure 5. Scanning electron micrograph (SEM) of chemical synthesized of (a) FA (b) PANI and (c-f) composite of polyaniline-flyash PANI-FA (0.02), PANI-FA (0.1), PANI-FA (0.5) and PANI-FA (1.0), EDXA spectra from (g-i) composite of polyaniline-flyash PANI-FA (0.1), PANI-FA (0.5) and PANI-FA (1.0).

dine base form of PANI significantly changes the aggregate state of polymeric molecular chain. The incorporation of metal oxides into the polymeric network induces uniform porosity and is expected to be advantageous for gas and biosensing applications.

\section{Conclusions}

In this study we demonstrated a way to use the toxic waste like fly ash (FA) to enhance the characteristic properties of polyaniline (PANI). Polyaniline fly-ash (PANI-FA) composites were prepared by oxidative polymerization of aniline with fly ash in presence of ammonium persulphate. The morphology of new composite materials was studied by scanning electron microscopy (SEM). SEM and EDXA pictures have been confirmed the complexation of metal oxide with emaraldine base form of PANI, significantly changing the aggregate state of polymeric molecular chain. The increase of kinetic energy as seen from TGA for the PANI-FA composite compared to PANI and FA indicate strong complexation of metal oxide with PANI emaraldine base.

\section{Acknowledgements}

Raju Khan is thankful to the Department of Science \& Technology (DST), Govt. of India, for financial support under the Young Scientist Scheme project No. SR/FTP/CS-77/2007.

\section{References}

[1] R. L. N Chandrakanthi and M. A. Careem, "Preparation and Characterization of $\mathrm{CdS}$ and $\mathrm{Cu} 2 \mathrm{~S}$ Nanoparticle/Polyaniline Composite Films," Thin Solid Films, Vol. 417, 2002, pp. 51-56. doi:10.1016/S0040-6090(02)00600-4

[2] J. E. Albuquerque, L. H. C. Mattoso, D. T. Balogh, R. M. 
Faria, J. G. Masters and A. G. MacDiarmid, "A Simple Method to Estimate the Oxidation State of Polyanilines," Synthetic Metals, Vol. 113, 2000, pp. 19-22. doi:10.1016/S0379-6779(99)00299-4

[3] D. C. Schnitzler, M. S. Meruvia, I. A. H. Mmelgen and A. J. G. Zarbin, "Preparation and Characterization of Novel Hybrid Materials Formed from (Ti,Sn)O2 Nano-Particles and Polyaniline," Chemistry of Materials, Vol. 15, No. 24, 2003, pp. 4658-4665. doi:10.1021/cm034292p

[4] F. Huguenin, G. M. Janete, E. A. Ticianelli and R. M. Torresi, "Structural and Electrochemical Properties of Nanocomposites Formed by V2O5 and Poly (3-Alkylpyrroles)," Journal Power Sources, Vol. 103, 2001, pp. 113-136. doi:10.1016/S0378-7753(01)00851-5

[5] V. A. Samoylov, Q. Hao, M. Y. Shirshov, C. Swart, E. Pringsheim, M. V. Mirsky and O. S. Wolfbeis, "Nanometer-Thick SPR Sensor for Gaseous HCl," Sensors \& Actuators, Vol. 106, 2005, pp. 369-372. doi:10.1016/j.snb.2004.08.029

[6] M. Ando, C. Swart, E. Pringsheim, M. V. Mirsky and O. S. Wolfbeis, "Optical Ozone-Sensing Properties of Poly (2-Chloroaniline), Poly (N-Methylaniline) and Polyaniline Films," Sensors \& Actuators B, Vol. 108, 2005, pp. 528-534. doi:10.1016/j.snb.2004.12.083

[7] W. Schultze and H. Karabalut, "Electrochemical Copolymerization of M-Toluidine and O-Phenylenediamin," Electrochimica Acta, Vol. 50, 2005, pp. 1739-1745. doi:10.1016/j.electacta.2004.10.023

[8] H. Bai and G. Shi, "Gas Sensors Based on Conducting Polymers," Sensor, Vol. 2, 2007, pp. 267-307. doi:10.3390/s7030267

[9] B. D. Malhotra, A. Chaubey and S. P. Singh, "Prospects of Conducting Polymers in Biosensors," Analytica Chimica Acta, Vol. 578, 2006, pp. 59-74.

[10] D. C. Trivedi, "Handbook of Organic Conductive Molecules and Polymers," Wiley, Chichester, 1997, pp. 505572.

[11] R. Khan and M. Dhayal, "Chitosan/Polyaniline Hybrid Conducting Biopolymer Base Impedimetric Immunosensor to Detect Ochratoxin-A," Biosensors \& Bioelectronics, Vol. 24, 2009, pp.1700-1705. doi:10.1016/j.bios.2008.08.046

[12] R. Khan, A.Kaushik and A. P. Mishra, "Immobilization of Cholesterol Oxidase onto Electrochemically Polymerized Film of Biocompatible Polyaniline-Triton X-100," Materials Science and Engineering: C, Vol. 29, 2009, pp. 1399-1403. doi:10.1016/j.msec.2008.11.001

[13] R. Khan, P. R. Solanki, A. Kaushik, S. P. Singh, S.Ahmad and B. D. Malhotra, "Cholesterol Biosensor Based on Electrochemically Prepared Polyaniline Conducting Polymer Film in Presence of a Nonionic Surfactant," Journal of Polymer Research, Vol. 16, 2009, pp. 363-373. doi:10.1007/s10965-008-9237-8

[14] A. Ansari, R. Khan, K. N. Sood and B. D. Malhotra, "Polyaniline-Cerium Oxide Nanocomposite for Hydrogen Peroxide Sensor," Journal of Nanoscience \& Nanotechnology, Vol. 9, 2009, pp. 4679-4685. doi:10.1166/jnn.2009.1085
[15] A. Kaushik, J. Kumar, M. K. Tiwari, R. Khan, B. D. Malhotra, V. Gupta and S. P. Singh, "Fabrication and Characterization of Polyaniline - ZnO Hybrid Nanocomposite Thin Film," Journal of Nanoscience \& Nanotechnology, Vol. 8, 2008, pp. 1757-1761. doi:10.1166/jnn.2008.006

[16] A. Kaushik, R. Khan, V. Gupta, B. D. Malhotra and S. P. Singh, "Hybrid Cross-Linked Polyaniline -WO3 NanoComposite Thin Film Using Thermal Vacuum Deposition Technique for NOx Gas Sensing," Journal of Nanoscience \& Nanotechnology, Vol.9, 2009, pp. 1792-1796. doi:10.1166/jnn.2009.417

[17] D. Y. Godovsky, A. E. Vorfolomeer, D. F. Zaretskya and R. L. N. Chandrakanthi, "Preparation of Nanocomposites of Polyaniline and Inorganic Semiconductors," Journal of Material Chemistry, Vol. 11, 2005, pp. 2465-2469. doi: $10.1039 / \mathrm{b} 103048 \mathrm{p}$

[18] E. I. Iwuoha, S. E. Mavundla, V. S. Somerset, L. F. Petrik, M. J. Klink, M. Sekota and P. Bakers, "Electrochemical and Spectroscopic Properties of Fly Ash-Polyaniline Matrix Nanorod Composites," Microchimica Acta, Vol. 155, 2006, pp. 453-458. doi:10.1007/s00604-006-0584-Z

[19] C. A. Rees, J. L. Provis, G. C. Lukeya and J. S. J. van Deventer, "Attenuated Total Reflectance Fourier Transform Infrared Analysis of Fly Ash Geopolymer Gel Aging," Langmuir, Vol. 23, 2007, pp. 8170-8179. doi:10.1021/la700713g

[20] S. Sathiyanarayanan, S. S. Azim and G. Venkatachari, "Preparation of Polyaniline- $\mathrm{Fe}_{2} \mathrm{O}_{3}$ Composite and Its Anticorrosion Performance," Synthetic Metats, Vol. 157, 2007, pp. 751-757. doi:10.1016/j.synthmet.2007.08.004

[21] Y. Cao, S. Z. Li, Z. J. Xuea and D. Guo, "Spectroscopic and Electrical Characterization of Some Aniline Oligomers and Polyaniline," Synthetic Metats, Vol. 16, No. 3, 1986, pp. 305-315. doi:10.1016/0379-6779(86)90167-0

[22] J. Libert, J. Cornil, D. A. dos Santos and J. L. Bredas, “A Theoretical Investigation of from Neutral Oligoanilines to Polyanilines: The Chain-Length Dependence of the Electronic and Optical Properties," Physical Review B, Vol. 56, No. 14, 1997, pp. 8638-8650. doi:10.1103/PhysRevB.56.8638

[23] M. S. Cho, S. Y. Park, J. Y. Hwang and H. J. Choi, "Synthesis and Electrical Properties of Polymer Composites with Polyaniline Nanoparticles," Materials Science and Engineering: C, Vol. 24, 2004, pp. 15-18. doi:10.1016/j.msec.2003.09.003

[24] H.-J. Glasel, E. Hartmann and J. Hormes "Preparation of Barium Titanate Ultrafine Powders from a Monomeric Metallo-Organic Precursor by Combined Solid-State Polymerisation and Pyrolysis," Journal of Materials Science, Vol. 34, 1999, pp. 1-5. doi:10.1023/A:1004533926099

[25] G. D. La Puente, G. Marban and E. Fuente, "Modelling of Volatile Product Evolution in Coal Pyrolysis. The Role of Aerial Oxidation," Journal of Analytical and Applied Pyrolysis, Vol. 44, 1998, pp. 205-218. doi:10.1016/S0165-2370(97)00078-8

[26] A. Arenillas, F. Rubiera, J. J. Pis, M. J. Cuesta, M. J. Iglesias, A. Jimenez and I. Suarez-Ruiz, "Thermal Beha- 
viour during the Pyrolysis of Low Rank Perhydrous Coals," Journal of Analytical and Applied Pyrolysis, Vol. 68-69, 2003, p. 371. doi:10.1016/S0165-2370(03)00031-7

[27] A. W. Coats and J. R. Redfern, "Kinetic Parameters from Thermogravimetric Data," Nature, Vol. 201, 1964, pp. 68-69. doi:10.1038/201068a0
[28] M. V. Kok, E. Ozbas, O. Karacan and C. Hicyilmaz, "Effect of Particle Size on Coal Pyrolysis," Journal of Analytical and Applied Pyrolysis, Vol. 45, 1998, pp. 103110. doi:10.1016/S0165-2370(98)00063-1

[29] P. R. Soloman, M. A. Serio, R. M. Carangelo and J. R. Markham, "Very Rapid Coal Pyrolysis," Fuel, Vol. 65, 1986, pp. 182-194. doi:10.1016/0016-2361(86)90005-0 